\title{
MASONRY MACRO-BLOCK ANALYSIS
}

\author{
Nuno Mendes \\ ISISE, University of Minho, Guimarães, Portugal \\ nunomendes@civil.uminho.pt
}

Synonyms: Macro-block, macro-element.

\section{Introduction}

The structural analysis involves the definition of the model and selection of the analysis type. The model should represent the stiffness, the mass and the loads of the structure. The structures can be represented using simplified models, such as the lumped mass models, and advanced models resorting the Finite Element Method (FEM) and Discrete Element Method (DEM). Depending on the characteristics of the structure, different types of analysis can be used such as limit analysis, linear and non-linear static analysis and linear and non-linear dynamic analysis.

Unreinforced masonry structures present low tensile strength and the linear analyses seem to not be adequate for assessing their structural behaviour. On the other hand, the static and dynamic non-linear analyses are complex, since they involve large time computational requirements and advanced knowledge of the practitioner. The non-linear analysis requires advanced knowledge on the material properties, analysis tools and interpretation of results. The limit analysis with macro-blocks can be assumed as a more practical method in the estimation of maximum load capacity of structure. Furthermore, the limit analysis require a reduced number of parameters, which is an advantage for the assessment of ancient and historical masonry structures, due to the difficult in obtaining reliable data.

The observation of the damage in masonry buildings caused by earthquakes in the past has been shown that the masonry structures can be discretized into a several macro-blocks and interfaces. The macro-blocks are portions of a structure with similar material properties and structural behaviour, to which the mechanical properties of the material can be assigned or, by simplification, they can be assumed to be infinitely rigid. The interfaces in a macro-modelling represents, in general, the cracks associated to the failure mechanisms. However, in a micromodelling strategy applied to masonry, in which the units and the joints are individually considered, the interfaces simulate the behaviour of the joints. Furthermore, different criteria for the strength parameters of macro-blocks and interfaces can be considered.

The limit analysis (Figure 1) with macro-blocks is a simplified and powerful structural analysis tool to evaluate the ultimate capacity of masonry structures by static models, involving the equilibrium of the macro-blocks through of the limit analysis basic concepts. 


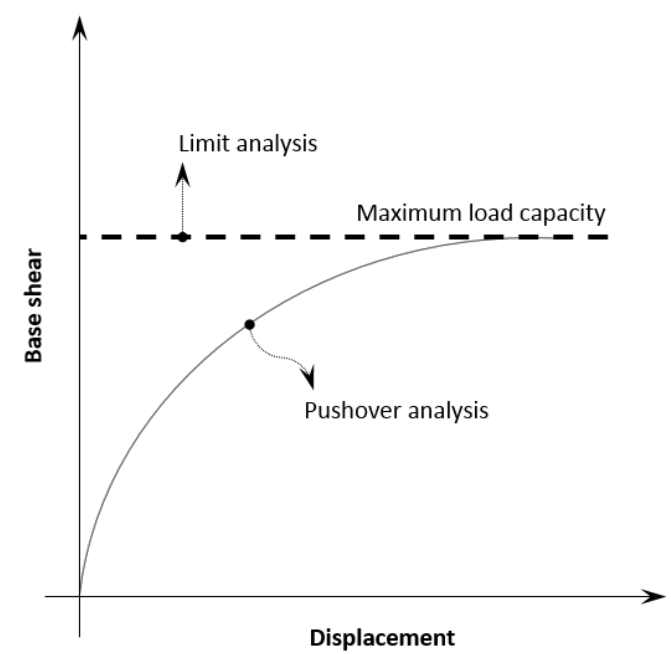

Figure 1 - Maximum load capacity obtained from the pushover analysis and limit analysis.

\section{Classic limit analysis concepts}

In the classic limit analysis using rigid-plastic material behaviour, a yield function $\mathcal{T}$ based on stresses is defined and three states are considered (Figure 2): (i) if $T<0$ the material presents rigid behaviour and the material remains undamaged; (ii) if $\mathcal{T}=0$ the material becomes plastic and corresponds to the yield surface; (iii) if $\tau>0$ the stress state is inadmissible. The points inside or on the yield function corresponds to the states in which the stress can be sustained by the material and the points outside corresponds to stress states inadmissible for the material. The classic limit analysis considers that when the stress states is on the yield surface the material becomes plastic and flow direction is normal to the yield surface. This assumption is called as associated flow or normality condition. The associated flow implies that the yield surface must be convex and provides the maximum energy dissipation (Nielsen 1999).
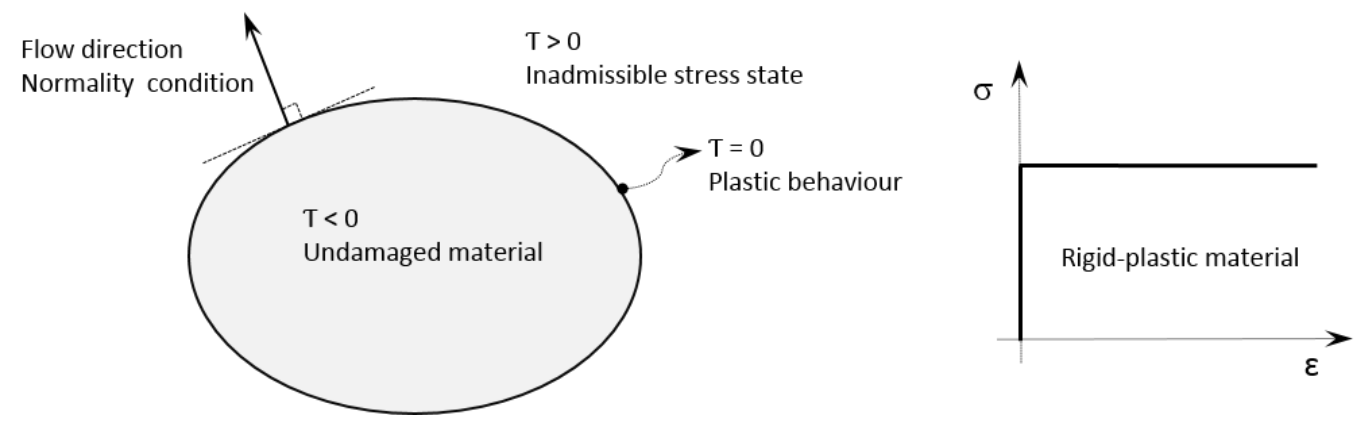

Figure 2-Classic limit analysis theory.

A general load on a structure can be multiplied by a load factor $\delta$ and increased from zero to a limit (failure load factor $\delta_{F}$ ) for which the structure remains the equilibrium condition (equilibrium between internal and external forces) and the yield condition (the stresses must be less than or equal to the material strength), i.e. the structure remains safe. This approach is associated to the static or lower bound theorem of the classic limit analysis, in which the safety factor is the largest of all the statically admissible load factors $\left(\delta_{L}\right)$. Furthermore, the classic limit analysis presents the kinematic approach, in which a sufficient number of releases (e.g. plastic hinges) have to occur to transform the structure into a mechanism. Each kinematically 
admissible mechanism presents an associated load factor, which is, in general, larger or equal to the safety factor. This approach corresponds to the kinematic or upper bound theorem of the classic limit analysis, in which the safety factor is the smallest of all kinematically admissible load factors $\left(\delta_{u}\right)$. The largest load factor obtained from the static theorem and the smallest load factor obtained from the kinematic theorem are the same, stating that a structure can present a statically admissible state and be unsafe if a sufficient number of sections reach the yield surface to transform the structure into a mechanism. This particular case corresponds to the uniqueness theorem of the classic limit analysis. The graphical representation of the theorems of classic limit analysis is presented in Figure 3 and are summarized as follows (Kamenjarzh 1996; Nielsen 1999):

- Static or lower bound theorem: If a load presents a magnitude such that the stress state satisfies the equilibrium and yield conditions, then the structure will not collapse and the load factor $\left(\delta_{L}\right)$ is less than or equal to failure load factor $\left(\delta_{F}\right)$. In this static approach the failure load factor is determined by searching the for the maximum load factor.

- Kinematic or upper bound theorem: If a kinematic admissible mechanism can be found for which the work of the external loads exceeds the internal work, then the structure will collapse and the load factor $\left(\delta_{U}\right)$ is greater than or equal to the failure load factor $\left(\delta_{F}\right)$. In the kinematic approach the failure load factor is determined by searching for the minimum load factor.

- Uniqueness theorem: If the equilibrium, mechanism and yield conditions are satisfied, then the load factor obtained from the static and kinematic approach are the same and is equal to the failure load factor $\left(\delta_{F}\right)$. Thus, the failure load factor is determined by equating the load factors of both approaches and using for example optimization techniques.

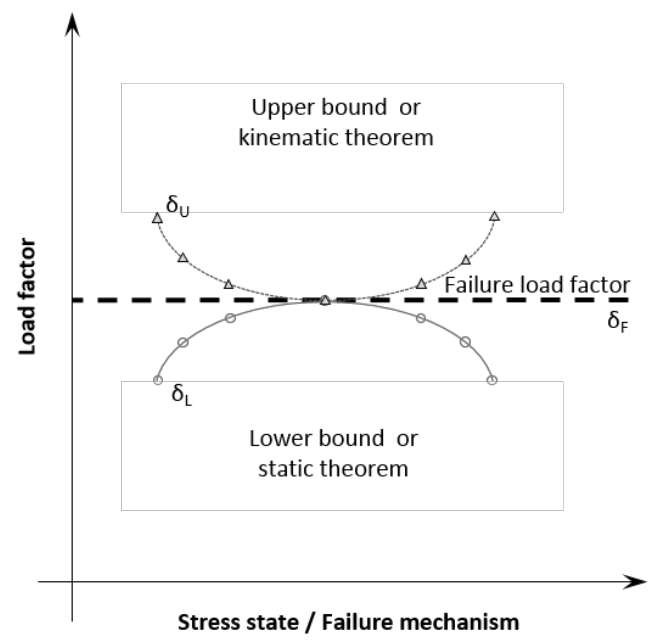

Figure 3-Graphical representation of the limit analysis theorems.

\section{Application of limit analysis in masonry structures}

Heyman (1966) was among the first and the most famous promoters to use the limit analysis based on plasticity theory on arches and other masonry structures. Although the masonry is a quasi-brittle material, the plastic limit analysis was used to assess the structural behaviour of masonry structures, assuming the flowing assumptions: 
- Masonry has no tensile strength: Masonry presents low tensile strength and quasi-brittle tensile behaviour, mainly in ancient masonry structures built with weak mortar, justifying this assumption.

- Masonry has infinite compressive strength: In general, masonry presents a compressive strength higher than the compressive stresses present in the structure. For this reason, the most cases of collapse of masonry buildings are related to tensile and shear failures. Thus, this assumption is in general accepted and the crushing failure of masonry is not considered.

- Sliding failure is not permitted: Although the sliding between units can sometimes occur at the joints, in general the sliding failure is not the most relevant failure mechanism and this assumption can be accepted.

The failure load assessment of masonry structures based on the limit analysis and the assumptions defined by Heyman (1966) can be carried out using two approaches: (a) static approach; (b) kinematic approach.

In the static approach the resultant of the compressive stresses is plotted at each cross-section of the structure. The line containing all the positions of the compressive stress resultants is called by thrust line (Figure 4a). The thrust line can be determined by analytical methods or graphical methods, such as funicular polygon (Kooharian 1952). The structure is safe when the thrust lin is located totally inside of the geometry of structure and the equilibrium with the external loads can be found. In this conditions and according to the lower bound theorem, the applied load is less than or equal to the failure load. Nevertheless, several thrust lines contained in the geometry of the structure can be found, meaning that the thrust line definition is an indeterminate problem (hyperstatic structure), where a solution can be obtained by using an iterative method. Thus, if the thrust line is found to be totally inside of the geometry of structure was found, the structure is safe, which it does not mean that the structure necessarily works according to this solution. However, nothing can be concluded with respect to the stability if the thrust line is outside of the geometry of structure, unless the infinite possibilities were tried.

Regarding to kinematic approach, when the structure presents an enough number of hinges to transform it into a mechanism (Figure $4 \mathrm{~b}$ ), the load factor obtained by equating the work of the external loads to zero corresponds to an upper bound limit (upper bound theorem). The load obtained from the kinematic approach is greater than or equal to the failure load. Different positions for the hinges on the structure can be adopted and several mechanism, and respective load factors, can be found. The mechanism that presents the lowest load factor corresponds to the collapse mechanism. Thus, the kinematic approach presents also several solutions.

Taking into account the results obtained from both approaches, the static approach presents a safe solution of the maximum load capacity of masonry structures. However, for complex masonry structures it can be difficult to predict the position of the thrust line. On the other hand, the kinematic approach can lead to an unsafe estimation of the ultimate load. This problem can be solved by implementing computational tools of optimization and searching by the failure load (minimum load factor).

A particular case can be occur when the thrust line becomes tangent to the boundaries of the geometry at an enough number of cross-sections to create a mechanism (Figure 4c). In this conditions, the mechanism corresponds to the collapse mechanism, the thrust line is unique and the load corresponds to the failure load (uniqueness theorem).

The assumptions adopted for the limit analysis of masonry structures are simplifications of its real and complex structural behaviour. In fact, the compressive strength of masonry is not infinite and the structures can present high concentrations of compressive stress. The use of 
finite compressive strength for masonry is more realistic and can provide a small reduction of the load factor at the collapse. Furthermore and as previously mentioned, the sliding failure can occur, which can be a non-conservative assumption for some particular construction techniques of masonry (e.g. sliding at the interface between the rings of a multi-ring masonry bridge). The consideration of the sliding failure implies the lack of the normality condition (non-associated flow). Thus, several researchers have been conducted efforts to develop limit analysis considering the crushing, the sliding and twisting failures of masonry. For more information on innovate limit analyses of masonry structures see e.g. Orduña and Lourenço (2003), Gilbert et al. (2006) and Portioli et al. (2013).

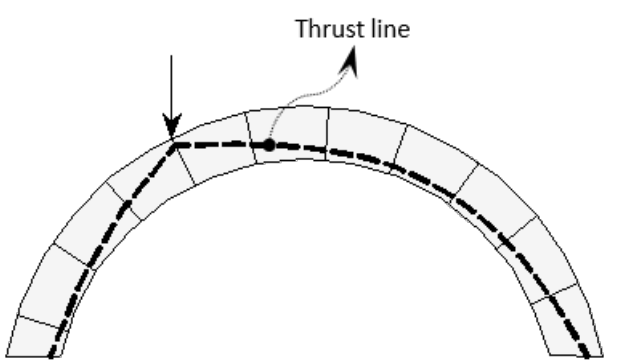

(a)

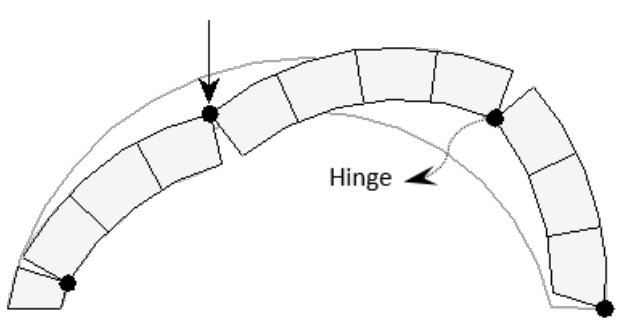

(b)

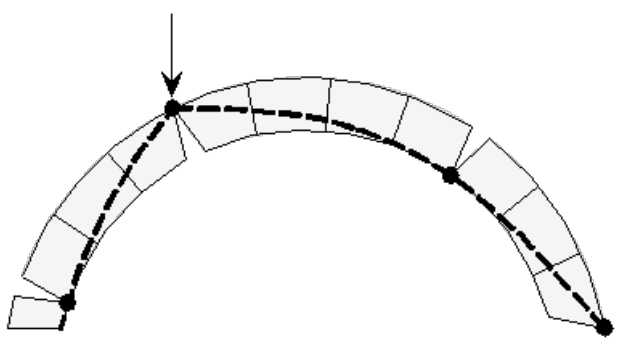

(c)

Figure 4-Application of the limit analysis to a masonry arch: (a) Static approach (lower bound theorem); (b) Kinematic approach (upper bound theorem); (c) Uniqueness theorem.

\section{Macro-block approach}

The limit analysis with macro-blocks allows to determine the maximum load capacity of structures by using simple procedures based on kinematic approach and involving the equilibrium of the macro-blocks. A macro-block corresponds to a portion of structures with similar material properties and structural behaviour, which can represent the structural element (e.g. piers) or a set of structural elements (e.g. a façade). In this approach, the structure is discretized in several macro-blocks with independent structural behaviours. This type of analysis is a practical tool for assessing of the structural behaviour of structures and does not require a high number of parameters for the material properties.

The existing masonry structures presents in general local modes under an earthquake, due to the loss of equilibrium of parts of the structure. Thus, the limit analysis using macro-blocks is particularly suitable for evaluating the seismic performance of existing masonry buildings, considering even the in-plane or the out-of plane collapse mechanisms (Figure 5). It should be noted that the out-of-plane behaviour of masonry structures is complex and that in general this typology of buildings do not satisfy the assumptions of the typical static non-linear analysis imposing lateral forces proposed for reinforced concrete buildings (rigid floors, regularity in-plan and in-elevation, and response governed by the first mode). Furthermore, this type of analysis 
is also a useful tool for evaluating the efficiency of strengthening techniques applied to existing masonry buildings.

In general, the application of limit analysis with rigid macro-blocks for masonry structures is based on the assumptions proposed by Heyman (1966) (no tension strength, infinite compressive strength and the sliding cannot occur). However, more realist assumptions can be considered, such as a finite compressive strength.

The macro-block approach involves a first step for selection of mechanisms. The mechanisms can be proposed on the basis of the knowledge obtained from the post-earthquake survey of similar buildings, using the crack patterns obtained from the experimental research and on the basis of the practitioner experience. The selection of the mechanisms to consider is a fundamental step in this type of analysis and requires a detailed knowledge of the features of the structure, such as the quality of the connections between masonry and the connections between masonry walls and floors, the presence of cracks and the interaction with adjacent buildings. In fact, a bad evaluation of the possible mechanisms can lead to the non-consideration of the mechanism with the lowest load factor and, consequently, can lead to a failure load higher than the real maximum capacity of the structure. However, the main local collapse mechanisms of masonry buildings typologies have been compiled in abacus on the form of graphical interpretations schemes, such the abacus for religious and urban masonry buildings (D'Ayala and Speranza 2002, Lagomarsino and Podesta 2004). Next, the load factor of each considered mechanism is determined applying the principle of virtual works and the mechanism that presents the lowest load factor is assumed as the collapse mechanism. Finally, the stability of the structure is analysed taking into account the load factor of the collapse mechanism and the requirements defined in the codes (e.g. by comparing of the maximum capacity in terms of horizontal acceleration on the structural and the demands in terms of Peak Ground Acceleration $(P G A))$.

Subsequently, an example of application of the limit analysis with rigid macro-blocks for masonry structures is presented.

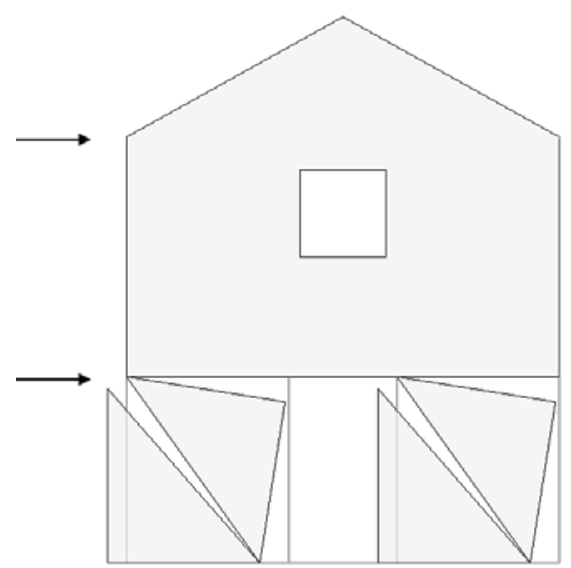

(a)

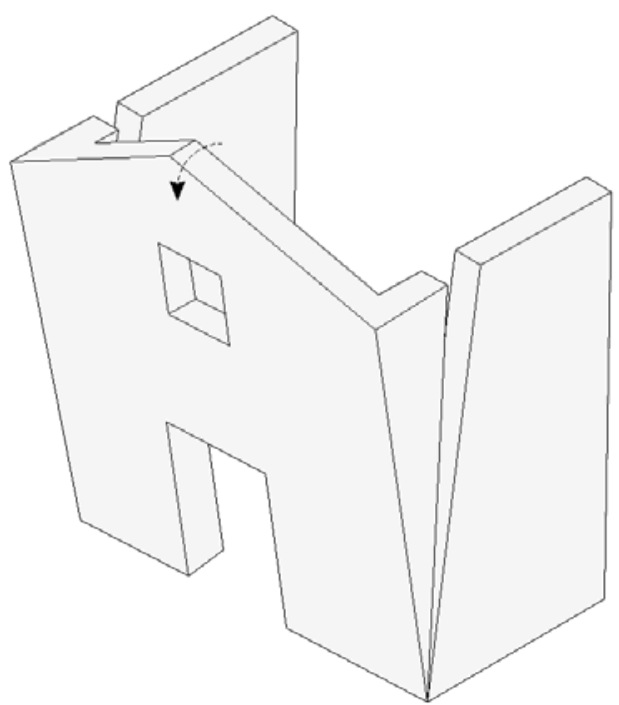

(b)

Figure 5 - Examples of collapse mechanisms using macro-blocks: (a) In-plane collapse of façade; (b) Out-of-plane collapse of façade. 


\subsection{Example of application}

Figure 6a presents a possible out-of-plane mechanism of a façade from masonry buildings with ties at the top and subjected to a seismic action with uniform acceleration acting in the orthogonal direction to the plan of the façade. The structure presents three horizontal cracks at the façade and two cracks at the corners. This local mechanism is composed by three hinges, in which the hinge associated to the crack within the wall is not initially known. Figure $6 \mathrm{~b}$ shows the scheme of the mechanism in analysis. The height $H$ and thickness $T$ of the masonry wall are equal to $3.50 \mathrm{~m}$ and $0.30 \mathrm{~m}$, respectively. The specific mass of the masonry $r$ is equal to 20 $\mathrm{kN} / \mathrm{m}^{3}$. Furthermore, it assumed that the vertical force at the top of the masonry wall $N$ is equal to $10 \mathrm{kN} / \mathrm{m}$.

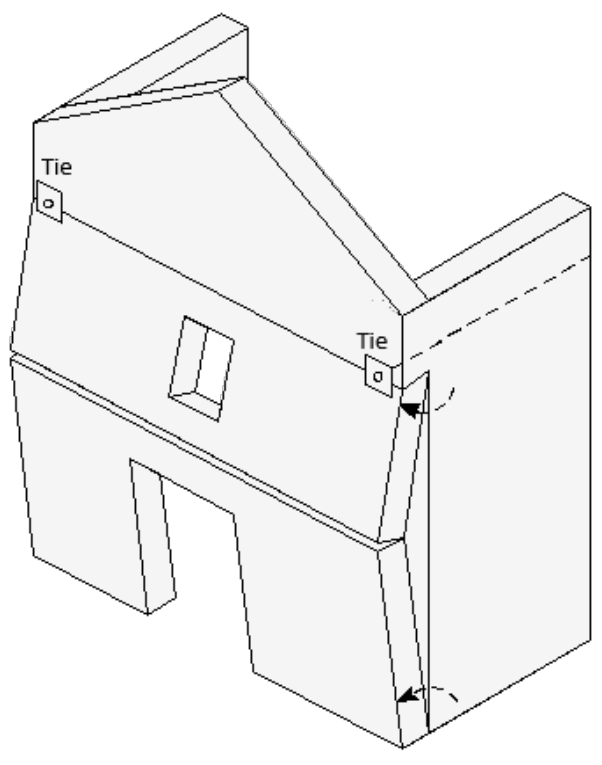

(a)

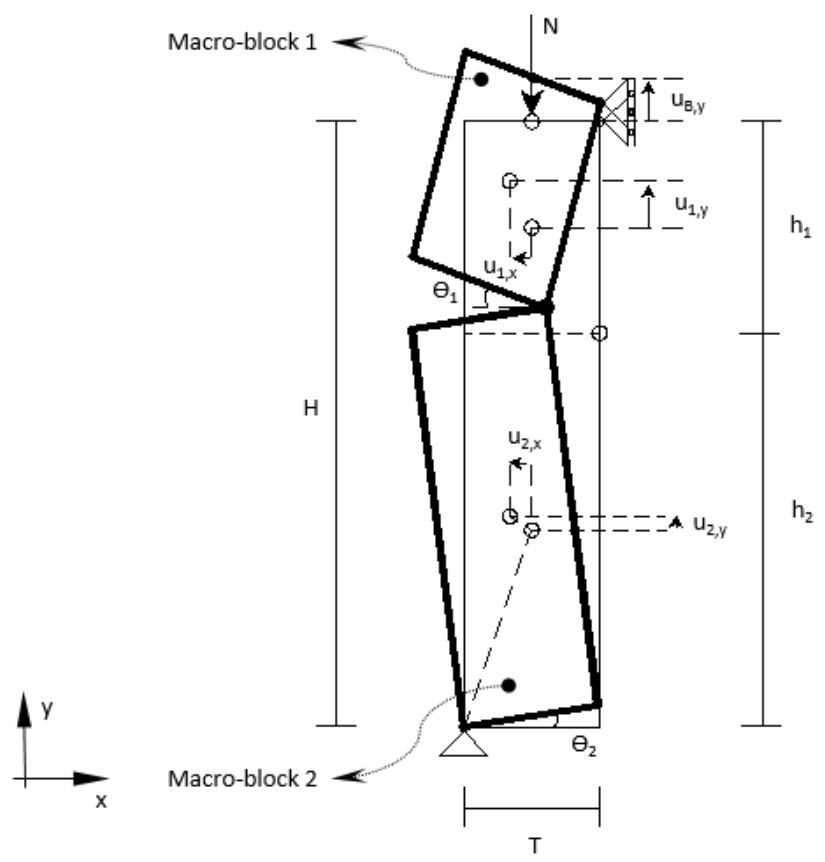

(b)

Figure 6-Out-of-plane collapse of tied masonry wall: (a) general view; (b) scheme of the mechanism.

The first step corresponds to the determination of the variables of rotation $(\theta)$ and displacements of the macro-blocks at theirs centre of mass $(u)$ in the vertical and horizontal directions. Since the position of the crack within the wall is not known, the heights of the macroblocks are defined as a function of the variable $X$ :

$$
\begin{gathered}
h_{1}=X H=3.5 X \\
h_{2}=(1-X) H=(1-X) 3.5
\end{gathered}
$$

If a rotation $\theta_{1}=1$ is given to macro-block 1 , then the macro-block 2 rotation is:

$$
\begin{gathered}
\theta_{1} h_{1}=\theta_{2} h_{2} \\
\theta_{2}=\frac{h_{1}}{h_{2}} \theta_{1} \\
\theta_{2}=\left(\frac{X}{1-X}\right) \theta_{1}=\frac{X}{1-X}
\end{gathered}
$$


and the displacements of the macro-blocks and of application of the vertical force $N$ are:

$$
\begin{gathered}
u_{1, x}=\frac{h_{1}}{2} \theta_{1}=\frac{X H}{2}=\frac{3.5}{2} X \\
u_{1, y}=\frac{T}{2} \theta_{1}+T \theta_{2}=\frac{T}{2}+T \frac{X}{1-X}=0.15+0.30 \frac{X}{1-X} \\
u_{2, x}=\frac{h_{2}}{2} \theta_{2}=\frac{(1-X)}{2} H \frac{X}{(1-X)}=\frac{X H}{2}=\frac{3.5}{2} X \\
u_{2, y}=\frac{T}{2} \theta_{2}=\frac{T}{2} \frac{X}{(1-X)}=0.15 \frac{X}{(1-X)} \\
u_{B, y}=u_{1, y}=\frac{T}{2}=0.15+0.30 \frac{X}{1-X}
\end{gathered}
$$

The forces applied at the macro-blocks due to the self-weight $(P)$ and the inertial forces proportional to mass $(F)$ are:

$$
\begin{gathered}
P=\gamma T H=21 \mathrm{kN} / \mathrm{m} \\
P_{1}=P X=21 X \\
P_{2}=P(1-X)=21(1-X) \\
F=\delta P=21 \delta \\
F_{1}=\delta P_{1}=21 X \delta \\
F_{2}=\delta P_{2}=21(1-X) \delta
\end{gathered}
$$

where $\delta$ is the load factor. $P$ and $F$ are forces by meter of wall width.

The application of the principal of virtual works provides:

$$
\begin{gathered}
-P_{1} u_{1, y}-P_{2} u_{2, y}-N u_{B, y}+F_{1} u_{1, x}+F_{2} u_{2, x}=0 \\
-\left(3.15 X+\frac{6.3 X^{2}}{1-X}\right)-(3.15 X)-\left(1.5+\frac{3 X}{1-X}\right)+\left(36.75 X^{2} \delta\right)+ \\
\left(36.75 X \delta-36.75 X^{2} \delta\right)=0 \\
-6.3 X-\frac{\left(3 X+6.3 X^{2}\right)}{1-X}-1.5+36.75 X \delta=0
\end{gathered}
$$

Thus, it is possible to obtain the load factor as a function of $X$ :

$$
\delta=\frac{6.3 X+\frac{\left(3 X+6.3 X^{2}\right)}{1-X}+1.5}{36.75 X}
$$

The minimum load factor $\delta$ can be computed by equating the:

$$
\frac{d \delta}{d x}=0
$$


and a value of $X$ equal to 0.287 is obtained. Replacing the value of $X$ in the Eq. (16), the minimum load factor is equal to 0.50 , leading to the conclusion that position of the crack within of façade that cause the minimum load factor is equal to $2.50 \mathrm{~m}$ with respect to the base of structure $\left(h_{2}=2.50 \mathrm{~m}\right)$.

\section{Summary}

The limit analysis with macro-blocks is a simple tool for evaluating the maximum load capacity of masonry structures. The possible mechanisms are proposed and then the respective load factors are determined. The mechanism that presents the minimum load factor corresponds to the collapse and its load factor is assumed as the failure load. Thus, a careful evaluation of the possible mechanisms is needed, aiming at not excluding any important mechanism and predicting correctly the maximum load capacity of the structure.

The macro-block analysis applied to masonry structures is based on the first assumptions used for the classic limit analysis of arches (no tension strength, infinite compressive strength, the sliding cannot occur), which is acceptable for the majority of the masonry buildings. However, several approaches considering more realistic assumptions have been proposed and implemented in computational tools, aiming at improving the capabilities and the applicability of this type of analysis.

As final conclusion, the limit analysis with macro-blocks is a powerful tool for the seismic assessment of masonry structures, including the historical buildings and the out-of-plane behaviour. Furthermore, it allows evaluating the efficiency of strengthening techniques.

\section{Cross-references}

Pushover Analysis

$368981 \quad 212 / 212$

Nonlinear Dynamic Seismic Analysis $368986 \quad 176 / 17$

Masonry Structures, Overview $\quad 367792 \quad 156 / 156$

\section{References}

D'Ayala, D., \& Speranza, E. (2002). An integrated procedure for the assessment of the seismic vulnerability of historic buildings. $12^{\text {th }}$ European Conference on Earthquake Engineering, paper n. 561.

Gilbert, M., Casapulla, C., \& Ahmed H.M. (2006). Limit analysis of masonry block structures with non-associative frictional joints using linear programming. Computers \& Structures, 84, 873-887. doi:10.1016/j.compstruc.2006.02.005

Heyman, J. (1966). The stone skeleton. International Journal of Solids and Structures, 2 (2), 249-279.

Kamenjarzh, J. (1996). Limit Analysis of Solids and Structures. CRC Press, Inc.

Kooharian, A. (1952). Limit analysis of voussoir (segmental) and concrete arches. Journal of the American Concrete Institute, 24 (4), 317-328.

Lagomarsino, S. \& Podesta, S. (2004). Seismic Vulnerability of Ancient Churches: I. Damage Assessment and Emergency Planning. Earthquake Spectra, 20 (2), 377-394. http://dx.doi.org/10.1193/1.1737735

Nielsen, M. (1999). Limit analysis and concrete plasticity. Second edition, CRC Press LLC. 
Orduña, A., \& Lourenço, P.B. (2003). Cap model for limit analysis and strengthening of masonry structures. Journal of Structural Engineering, 129 (10), 1367-1375. http://dx.doi.org/10.1061/(ASCE)0733-9445(2003)129:10(1367)

Portioli, F., Casapulla, C., Cascini, L., D’Aniello, M., \& Landolfo, R. (2013). Limit analysis by linear programming of 3D masonry structures with associative friction laws and torsion interaction effects. Archive of Applied Mechanics, 83 (10), 1415-1438. doi:10.1007/s00419013-0755-4 